\title{
James M. Anderson, Daily Life During the French
} Révolution

Westport (Connecticut) et Londres, Greenwood Press, 2007, 269 p., ISBN-13: 978-0-313-33683-6, 130,95 \$.

Jean-Pierre Gross

\section{(2) OpenEdition Journals}

Édition électronique

URL : https://journals.openedition.org/ahrf/11416

DOI : 10.4000 /ahrf. 11416

ISSN : 1952-403X

Éditeur :

Armand Colin, Société des études robespierristes

\section{Édition imprimée}

Date de publication : 1 mars 2008

Pagination : 221-223

ISSN : 0003-4436

\section{Référence électronique}

Jean-Pierre Gross, "James M. Anderson, Daily Life During the French Révolution », Annales historiques de la Révolution française [En ligne], 351 | janvier-mars 2008, mis en ligne le 29 décembre 2009, consulté le 06 août 2021. URL : http://journals.openedition.org/ahrf/11416 ; DOI : https://doi.org/ 10.4000/ahrf.11416 
l'explication du développement historique. Toutefois, si l'entreprise est courageuse, elle n'est pas sans susciter quelques remarques, notamment du point de vue de la terminologie. Lauteur a largement recours à ces riches notions que sont justement les expressions " révolution bourgeoise ", "lutte des classes " et " bourgeoisie », mais qui malheureusement ne font l'objet d'aucune explication en profondeur permettant de retracer leur généalogie, leur sens chez Marx et leur sens dans le discours historiographique sur la Révolution française. Par ailleurs, Henri Heller a visiblement négligé la dimension internationale de la Révolution française, y compris dans son analyse économique qui semble ignorer les problématiques coloniales. Mais ce ne sont là que détails qui ne doivent pas nous faire oublier qu'en remettant au centre de la réflexion historiographique des thèmes et des questions aussi importants que le rapport entre révolution et capitalisme, Henri Heller nous offre des perspectives de recherche renouvelées.

Julien LOUVRIER

James M. Anderson, Daily Life During the French Revolution, Westport (Connecticut) et Londres, Greenwood Press, 2007, 269 p., ISBN-13: 978-0-31333683-6, 130,95\$.

Déjà auteur d'une histoire du Portugal (2000) et d'une vie quotidienne pendant l'Inquisition espagnole (2002), ce professeur émérite de l'Université de Calgary au Canada n'est pas à son premier coup d'essai en matière d'histoire sociale. Sans être lui-même spécialiste de l'histoire de France, soulignons que James Anderson, en composant sa fresque, a eu recours aux travaux les plus récents d'historiens de la période révolutionnaire : qu'il suffise de mentionner les noms de Colin Jones, Christine Hillam ou John Frangos, pour ce qui est de l'histoire de la médecine et des hôpitaux, ou encore ceux de Gwynne Lewis, John Lough et David Garrioch, en ce qui concerne l'histoire sociale proprement dite. De manière plus générale, il a su intégrer dans son tableau l'éclairage un tant soit peu contestataire de Lynn Hunt, Suzanne Desan ou David Andress. Louvrage de James Anderson est par conséquent solidement documenté.

Comme on le sait, les récits de la "vie quotidienne " constituent un genre reconnu et consacré de l'historiographic anglo-saxonne, s'inscrivant dans la lignée de l'ouvrage classique de G. M. Trevelyan, English Social History (première édition parue en 1944). Genre souvent exploité avec panache en France même, qu'il suffise d'évoquer le chef-d'œuvre du regretté Henri Amouroux, La grande histoire des Français sous l'occupation. Pour ce qui est de 1789, l'ouvrage déjà ancien de Jean Robiquet sur la vie quotidienne sous la Révolution méritait une mise à jour, Anderson s'en est inspiré, en y apportant des compléments très riches issus de la recherche moderne. Il n'en reste pas moins que l'auteur se trouve confronté à une véritable gageure, en raison même de son approche du sujet. Face à une Révolution française faite d'incessants rebondissements, dont il ne ressort a priori aucune trame ćvolutive cohérente, et dont l'incidence immédiatc sur la vie des citoyens n'est pas aisée à déterminer, Anderson s'efforce de relever un triple défi : la nécessité de nous raconter l'Ancien Régime, afin de nous faire comprendre ce que la Révolution a balayé, puis de nous parler de la suite ; car, comme il l'affirme dans sa 
préface, afin d'en mieux dégager le quotidien vécu, il ambitionne d'englober les trois volets indissociables de son sujet : l'avant, le pendant et l'après (page IX). Ainsi chaque chapitre reprend cette démarche tripartite : qu'il soit consacré à l'économie, à la mode vestimentaire, aux spectacles, à la médecine, à la religion, aux femmes, à la vie urbaine, rurale ou militaire. Le lecteur non-initié risque de se sentir dérouté, et même aux yeux d'un historien aguerri, cet imbroglio chronologique à répétition finit par générer un grand flou.

La méthode choisie laisse donc à désirer. On aurait pu imaginer une approche plus traditionnelle, moins itérative, et fondée sur de solides repères, par exemple un livre divisé en quatre parties : $1^{\circ}$ la crise de l'Ancien Régime, $2^{\circ}$ l'euphorie des États génćraux et les promesses de $1789,3^{\circ}$ le basculement dans la guerre et l'avènement de la Terreur, $4^{\circ}$ le Directoire, puis la montée en puissance du Premier consul. Scénario logique plus propre à nous faire vivre l'expérience des Français face aux bouleversements, et mieux appréhender l'incidence du politique sur le vécu. Car le livre d'Anderson se veut à la fois une fresque, retraçant les grandes espérances et les grands retournements de l'époque révolutionnaire, mais parallèlement un récit de vies saisies dans leur humble quotidienneté et dans leur singularité ; de ce patchwork composé d'innombrables drames particulicrs, il cherche à façonner une mosaïque, dont chaque pièce devrait constituer une facette nécessaire de l'image. Il en résulte plutôt un puzzle défait, où la monarchie, la république et l'empire naissant se rencontrent pêle-mêle, et dont on a du mal à recombiner les éléments pour découvrir enfin le tableau d'ensemble.

C'est regrettable, d'autant plus que les sources exploitées par Anderson, souvent par spécialistes interposés, sont impeccables. Quand il leur reste fidèle, plutôt que de s'adonner à des récapitulatifs, dès qu'il cite l'expérience directe d'un témoin, le livre s'anime et rejoint le modèle historiographique forgé par Trevelyan : qu'il s'agisse d'Arthur Young, vedette parmi les voyageurs étrangers sillonnant la France à l'aube de la Révolution; ou de la comtesse de Carlyle, dix ans plus tôt, assistant à la grande foire de Beaucaire ; ou encore du romancier George Smollett, dans les années soixante, pourfendeur de la rouerie des mauvais aubergistes et de la vénalité des préposés des douanes. On découvre alors l'ancien royaume de France, cueilli sur le vif, tout comme, sous la plume de Trevelyan, on retrouvait l'Angleterre des Stuart et les ravages de la guerre civile à travers la vie mouvementée de Francis Russell, quatrième comte de Bedford. Toute anecdote véridique peut éclairer de sa lumière le quotidien d'une époque, mais revêt une signification plus parlante encore si elle dépasse le pittoresque pour illustrer soit un changement, soit une continuité. Tel le récit des innovations apportées aux soins des blessés sur le champ de bataille par le chirurgien Dominique-Jean Larrey, pionnier des premiers secours et des moyens d'évacuation, que James Anderson nous décrit de façon détaillée et convaincante (p. 214-216). Ici, la réalité de l'expérience militaire surgit à nos yeux, et l'on aurait souhaité davantage de comptes rendus exhaustifs de ce genre, qu'on découvre ça et là, comme des perles.

Malgré ces réserves, l'ouvrage de James Anderson servira de livre de référence fiable pour l'étudiant et le profane, qui lui seront redevables d'un tableau chronologique des années 1770 à 1799, d'une cartographie comparative, d'analyses bibliographiques succinctes en fin d'ouvrage, ou encore d'un glossaire sommaire des termes usités en français, destiné aux anglophones qui abordent la Révolution 
française pour la première fois. Regrettons que l'iconographie, assez variée et indispensable dans un tel ouvrage, soit uniquement en noir et blanc, hormis la couverture du livre, qui nous montre, en lever de rideau prometteur, le siège de la Bastille en couleur sur fond rouge.

Jean-Pierre Gross

Pascal Dupuy et Claude Mazauric, La Révolution française, regards d'auteurs, Paris, Vuibert, 2005, 441 p., ISBN 2-7117-4435-3, $23 €$.

" Récit à voix multiple », " recueil [... pour] saisir le mouvement de la Révolution [...] découvrir presque un siècle et demi d'historiographie révolutionnaire ", ces brefs extraits de la quatrième de couverture donnent clairement le sens du projet mené à bien par deux auteurs dont on sait la forte complicité intergénérationnelle comme si, dans une certaine mesure, ils prolongeaient cux-mêmes la chaîne historiographique qu'ils ont voulu recomposer.

En fait l'ouvrage associe constamment trois genres que l'édition sépare ordinairement : le rappel de généralités sur le cours de la Révolution, ce mouvement évoqué plus haut ; les textes longuement restitués d'auteurs, de la fin du XVIII siècle au milieu du $X X^{\bullet}$, très divers dans leurs inscriptions chronologiques et idćologiques; des prolongements synthétiques sur l'historiographic plus contemporaine.

La démarche présente beaucoup d'intérêt et quelques inconvénients. Elle offre d'abord une approche très dynamique du processus révolutionnaire et illustre à merveille le fait essentiel que tout est sujet à débat dans le laboratoire révolutionnaire, ce qui évidemment contribue à l'intérêt que nous devons continuer à lui porter. En second lieu, l'ouvrage présente le grand mérite de rendre accessible, notamment pour les étudiants, des textes de notoriétés très différentes : certains sont de grands classiques de $\mathbf{M}^{*}$ de Staël, Michelet, Quinet, Tocqueville, Jaurès... ; d'autres sont connus mais leur citation vaut mise en exergue de dimensions majeures de la Révolution, parfois un peu minorées sous prétexte qu'elles sont évidentes, je songe par cxemple au beau texte de Cabet évoquant la Nuit du 4 août et ses suites équivoques (p. 43-44) ou, sur un autre registre, au magnifique poème de Rimbaud célébrant le peuple levé (p. 189-193), contrepoint à la stigmatisation de la " lie du peuple " par Taine (p. 184-186) ; mais on ne saurait négliger non plus des témoignages ou analyses par des personnalités moins célèbres qui proposent des éclairages tout à fait intéressants, par exemple les notes publiées en 1796 par ce collaborateur de L.S. Mercier, Fantin Désodoards sur le choc de la fuite du roi et de Varennes (p. 163) ou les évocations du climat politique et militaire au printemps et à l'été 1792 (p. 169-172), par le républicain Peyrat dans La révolution et le livre de $M$. Quinet (1866). Ces quelques exemples, les noms et les dates cités illustrent aussi les inconvénients de l'ouvrage : le suivi de ces écritures multiples de l'histoirc, au fil des pages, s'avère parfois un peu sinueux dans la succession de textes, de dates et de factures diverses et à cause du continuum d'écriture entre ceux-ci et les commentaires de P. Dupuy et C. Mazauric - et ce malgré l'usage de typographies différentes. Ce continuum, d'ailleurs, rend furtif la mise en contexte des extraits proposés et l'identification de leurs auteurs alors que, justement, l'un des intérêts de ces rappro- 